\title{
The systemic sclerosis patient in the COVID-19 era: the challenging crossroad between immunosuppression, differential diagnosis and long-term psychological distress
}

\author{
Martina Orlandi ${ }^{1}$ (D) Gemma Lepri ${ }^{1}$ (D) Cosimo Bruni ${ }^{1}$ (D) Yukai Wang $^{2} \cdot$ Alessandro Bartoloni $^{3}$. \\ Lorenzo Zammarchi $^{3}$ - Laura Cometi $^{1}$ - Serena Guiducci ${ }^{1}$ • Marco Matucci-Cerinic ${ }^{1} \cdot$ Silvia Bellando-Randone $^{1}$
}

Received: 7 May 2020 / Revised: 7 May 2020 / Accepted: 19 May 2020 / Published online: 8 June 2020

(C) International League of Associations for Rheumatology (ILAR) 2020

\begin{abstract}
COVID-19 is a world health emergency which may inevitably affect the management of a complex autoimmune disease such as systemic sclerosis (SSc). Several SSc patients are frail and, in this pandemic, need a careful protection. The COVID-19 infection might complicate the clinical scenario of interstitial lung disease (ILD) in SSc because it determines a severe pneumonia characterized by radiological features similar to SSc-ILD. The striking CT similarities between the 2 diseases make it difficult to distinguish a worsening of SSc-ILD from COVID-19-ILD superinfection. Moreover, other aspects, like isolation during lock down, may cause a significant psychological stress which will pile up on the already difficult contact with the patients for a routine check-up. Moreover, the drug shortage is a real problem in these times. For these reasons, the rheumatologist in daily clinical practice should carefully differentiate the possible COVID-19 infection in order to optimize the patient management. Therefore, the challenge in everyday life will be to achieve in due time the differential diagnosis as well as the long-term psychological impact.
\end{abstract}

Key Points

- SSc patients should be encouraged to continue their chronic therapy; in case of immunosuppressive therapy it must be discontinued for safety in case of COVID-19 infection.

- Psychological support must be guaranteed to every SSc patients.

- COVID-19 pneuminia is hard to distinguish from an interstitial lung disease due to SSc lung involvment.

- Data sharing is fundamental for an optimal managment of SSc patients during COVID-19 pandemia.

Keywords COVID-19 · COVID-19 pneumonia · Systemic sclerosis

Martina Orlandi

martina.orlandi@unifi.it

Gemma Lepri

gemma.lepri@unifi.it

Cosimo Bruni

cosimo.bruni@unifi.it

Yukai Wang

stzxyywyk@126.com

Alessandro Bartoloni

alessandro.bartoloni@unifi.it

Lorenzo Zammarchi

lorenzo.zammarchi@unifi.it

Laura Cometi

lauracometi@alice.it
Serena Guiducci

serena.guiducci@unifi.it

Marco Matucci-Cerinic

marco.matuccicerinic@unifi.it

Silvia Bellando-Randone

s.bellandorandone@gmail.com

1 Department of Experimental and Clinical Medicine, Department of Geriatric Medicine, Division of Rheumatology AOUC \& Scleroderma Unit, University of Florence, Florence, Italy

2 Rheumatology and Immunology Department, Shantou Central Hospital, Affiliated Shantou Hospital of SUN YAT-SEN University, Shantou, China

3 Department of Experimental and Clinical Medicine, Infectious and Tropical Diseases Unit, AOUC, University of Florence,

Florence, Italy 


\section{Introduction}

The 2019 novel coronavirus virus (COVID-19) is highly contagious and has raised world concern because of its high morbidity and mortality. The pandemic rapidly spread worldwide to the point that led the World Health Organization (WHO) to declare the pandemic emergency on March 11, 2020 [1]. The epidemiological situation is constantly evolving, and data updated in April count more than 2,883,603 confirmed cases of COVID-19, including 198,842 deaths [2]. The COVID-19 virus affects mainly the lower airway tract determining a severe pneumonia characterized by interstitial involvement.

Today, COVID-19 represents a health emergency that may inevitably involve also the management of a complex autoimmune disease such as systemic sclerosis ( $\mathrm{SSc}$ ) which is a chronic systemic autoimmune disease characterized by vascular damage, autoimmunity, and fibrosis [3]. At present, we do not know yet how much SSc patients may be susceptible to the virus and which may be the disease course in those infected with SARS-CoV-2. Up to now, the literature reported only one SSc case treated monthly with intravenous tocilizumab, who developed a very mild disease and who had a consequent delay in the treatment [4].

Nonetheless, the TC features of the lung interstitial disease in SSc (ILD-SSc) and of the COVID-19 pneumonia have similarities that may be clinically relevant and have specific implications that should be considered in taking care of SSc patients during the COVID-19 pandemic.

\section{Immunosuppression in SSC}

Usually, SSc patients should have a higher infectious risk than the general population because of autoimmune dysregulation and chronic immunosuppressant therapies. The use of synthetic and biologic disease-modifying drugs (DMARDs) is associated with a potential to further increase the incidence of serious infections but an active disease may be a significant factor favoring an increase in infection risk too. However, specific data suggesting that a drug-induced immunosuppressed state might predispose SSc patients to SARS-CoV-2 infections are not available. However, data from immunosuppressed transplant patients do not suggest a higher frequency of severe COVID-19 in this patient cohort [5]. In SSc patients, the maintenance of disease remission during a pandemic infection is important to prevent disease flares avoiding unnecessary visits and hospitalizations, all of which increase potential exposure to COVID-19.

Some evidence suggest that immunosuppressive drugs, such as tocilizumab and JAK kinase inhibitors, may be useful in controlling the evolution of COVID pneumonia to the fatal pulmonary complication known as cytokine release syndrome
(CRS) ("cytokine storm"). This evidence may therefore suggest that SSc patients should continue their immunosuppressive medications [6]. However, some modifications may be considered on a case by case basis as appropriate.

In COVID-19 patients, the pulmonary complications are due to an exuberant local inflammatory response with diffuse alveolar damage. Cytokines and chemokines play a key role in the immune response against viral infections, and their altered production has been demonstrated in both SARS and MERS coronavirus infections [7]. Such altered levels have been shown to be likely due to the low synthesis of antiviral cytokines such as interferons (IFN) $\alpha$ or $\beta$ and in concomitant increased levels of other pro-inflammatory cytokines/ chemokines, especially interleukin (IL)-1, IL-6, which have pathogenic consequences. Recent preliminary data reported high plasma levels of cytokines including IL-6, related to the severity and the prognosis of the disease with a clear implication for the occurrence of "cytokine storm". Tocilizumab (TCZ), an anti-IL-6 receptor antibody used to treat lung involvement in SSc patients, has been also used for treatment of CRS [8]. In COVID-19 pneumonia patients, TCZ infusion led to clinical and radiological improvement, suggesting that an anti-IL-6 strategy might potentially rescue the patient from evolution to respiratory distress syndrome.

As TCZ may be mainly used to treat COVID-19 severe pneumonia cases, its availability must be guaranteed for the treatment of SSc. For this reason, whenever possible, it would be preferable to use subcutaneous administration instead of intravenous TCZ to keep the patient away from the hospital and also to avoid troubles in case of a possible intravenous drug shortage.

In these patients, it is important to keep a follow up thus maintaining a tight control to verify the specific case by case clinical needs. In fact, the infection risk in SSc is also related to disease activity and a flare due to therapy discontinuation would confer a higher risk of infection and of corticosteroid use that are moderately recommended in SSc patients [9]. While the practitioner should always encourage patients to avoid social contacts and maintain a strict isolation, the reference centers should guarantee a telematic assistance to SSc patients in these weeks of COVID-19 outbreak when the routine outpatient clinics are closed [42].

The International Societies of Rheumatology have proposed a set of practical recommendations to improve the management of COVID-19 patients and to decrease the risk of acquiring this infection in rheumatic patients undergoing immunosuppressive therapies $[10,11]$.

If an SSc patient develops symptoms of COVID-19 confirmed by two tests, it is important to follow the guidelines suggested by the rheumatology community and immunosuppression should be paused for the duration of the infection. If the patient has ILD secondary to SSc, a rapid laboratory and 
radiological screening must be performed quickly to distinguish the ILD-SSc flare from the SARS-CoV-2 superinfection.

In SSc patients, Hydroxychloroquine (HCQ) is seldom used [12]. The antiviral properties of chloroquine (CQ) and HCQ have been recently confirmed in HIV [13] and SARSCoV-1 with promising results $[14,15]$. Some papers identified a possible effect of HCQ on COVID-19 [16-19] in vitro studies showing an antiviral activity toward the SARS-CoV-2. Otherwise, in vivo data available are scarce and prone to significant bias due to methodological limitations. There is presently no medium to long-term follow-up data to support this approach, and all the effects presented require clinical trial confirmation, most of which are already underway. On April 2020, ClinicalTrials.gov search for COVID-19 and HCQ shows 44 registered trials. However, on the basis of preliminary results from ongoing clinical trials, some countries have incorporated $\mathrm{CQ} / \mathrm{HCQ}$ into their treatment protocols for certain patients with COVID-19, despite a lack of sufficient evidence of effectiveness [20, 21]. The practical advice for SSc patients on chronic antimalarials is to maintain the treatment, considering its antiviral activity as well as the immunomodulatory rather than immunosuppressive effect. The potential shortage of HCQ for patients with SSc due to the redirection of supply toward treatment of COVID-19 is concerning. There are no good substitutes for antimalarials in terms of the favorable risk-benefit ratio for treatment of certain manifestations of SSc, and several companies have ramped up the production of HCQ to ensure no interruption of treatment for patients with autoimmune conditions.

\section{Interstitial lung disease}

Patients with chronic ILD may be more prone to develop a severe COVID-19 lung infection [22]. In fact, ILD is one of the most severe complications in SSc patients and it is well known that SARS-CoV-2 has a specific tropism for lower respiratory tract and the pulmonary interstitium. In ILD-SSc, the interstitial disease likely originates from a dysregulation of the systemic immune system. COVID-19 induces direct lung injury by involving angiotensin-converting enzyme- 2 receptors, with potential evolution to diffuse alveolar damage [23, 24]. In SSc, COVID-19 may overlap and complicate ILD-SSc determining an acute severe pneumonia characterized initially by radiological features that may be confused with those of SSc-ILD. Therefore, the radiologist and the rheumatologist should always liaise to understand if the ongoing ILD involvement is secondary to disease progression or is an early phase of COVID infection. At interstitial level, bilateral and subpleural involvement and presence of ground-glass opacities (GGO) with or without consolidations are the most frequent radiological modifications present in both diseases. In fact, SSc-ILD is characterized by bilateral, lower-lobe predominant GGO, reticulations, and, in advanced cases, honeycombing [25-28]. In SARS-CoV-2 infection, severe pneumonia is characterized by bilateral GGO evolving to consolidations with a peripheral and subpleural distribution diffusing at also upper lobes.

The clinical presentation of SSc-ILD and COVID-19 can be similar and clinically characterized by dyspnea, fatigue, and non-productive cough. During SARS-CoV-2 infection, these symptoms may be accompanied by fever and rapid respiratory function decrease [29-31]. The absence of fever should not lower the suspicion for a SARS-CoV-2 infection in symptomatic (conjunctivitis, dysgeusia, olfactory loss, diarrhea, cough, dyspnea, asthenia) patients with SSc, particularly in those on immunosuppression, in which fever response can be absent. In these patients, it is of paramount importance to test rapidly for SARS-CoV-2 infection, as respiratory symptoms such as dry cough and shortness of breath can be wrongly attributed to worsening of underlying ILD-SSc. Moreover, in the literature, a large number of asymptomatic COVID-19 patients are reported [32].

It should not be forgotten that despite these clinical and radiological similarities, the two diseases present a very different progression rate. In fact, COVID-19 has acute progression leading to respiratory failure and exitus in few weeks $[8$, 18] while ILD-SSc has a chronic-sub chronic evolution leading to mortality in several years $[33,34]$. At present, COVID19 diagnosis relies on epidemiological and clinical criteria and serological confirmation with real-time reverse transcriptase polymerase chain reaction (rRT-PCR) of respiratory secretions [35]. It is now well known that this method may also yield false-negative results (especially in early disease) and therefore should be repeated after some days [36-39]. In this scenario, chest HRCT has a central role in diagnostic algorithm, in particular in early disease phase, in patients asymptomatic or paucisymptomatic for COVID-19 infection, pending infectious disease tests, and false-negative laboratory tests. The striking HRCT similarities between the 2 diseases make it difficult to distinguish a worsening of SSc-ILD from COVID-19-ILD superinfection. Clearly, even if there is an ILD-SSc, the patient's preventive isolation should be implemented immediately in any case.

\section{Routine clinical visits and labor0atory workout}

Patients typically require regular follow up visits and lab work out to ensure the early detection of SSc worsening, to diagnose complications, and to monitor the efficacy and toxicity of immunosuppression. In agreement with local institutions, an option is the use of telephone/telemedicine visits to minimize contact exposures [42]. If a visit is necessary, use of appropriate personal protective equipment (masks, gloves etc) is mandatory as well as aeration of the visiting room. Timing visits become very important to minimize the presence of people in 
a room. If laboratory testing can be safely delayed, then consideration for this may be made based on good clinical judgment.

\section{Psychosocial implications}

In these challenging times, the psychological implications of the current pandemic should be carefully considered in SSc patients [40]. The impact of the media daily reports on loss of lives from COVID-19 is increasing worldwide, especially in patients that have comorbidities and are suffering of chronic diseases [41]. Moreover, it can be very distressing to hear about the potential shortage of drugs (Tocilizumab, HCQ) that patients already use to control SSc. Conflicting reports about both the increased risk of COVID-19 in immunosuppressed patients as well as a recent statement that patients with autoimmune disease may be protected from getting COVID-19 by HCQ are not yet confirmed by solid evidence-based data. Social distancing to prevent viral spreading may further add to the psychological distress. The economic consequences and loss of employment affecting our patients and their families can have further implications on patient's quality of life. It is important that physicians remain vigilant on these psychological, social, and economic implications that may affect the SSc population during the current COVID-19 pandemic. As we know, stress is a well-recognized trigger for flares of autoimmune diseases, leading to increase the need for medical interventions. To reduce the risk of contagion, hospitals have suspended routine visits for chronic diseases, except for emergencies or oncological conditions. This is an important fact because some SSc patients, likely the frailest among them, require frequent visits. For example, patients with active digital ulcers must undergo weekly wound evaluation. Taken all together, these facts may lead patients to loneliness and disorientation. The rheumatologist may help patients in various ways, giving a correct information about COVID-19 and about the real risk every patient incurs, encouraging continuing immunosuppression, and guaranteeing the communication with the doctor in case of need.

\section{Conclusion}

Patients must be confident on clinician's work and extremely cautious. They must be encouraged to maintain their therapies, prevent infection by avoiding social contacts, and pause immunosuppression in case of infection. Rheumatologists must implement aggressive measures to prevent exposure to SARSCoV-2 infection, maintain disease remission, and address psychosocial aspects to ensure the best possible outcomes for patients during this pandemic.

Although our considerations have no epidemiological background, surprisingly, the number of reported SSc patients infected by COVID-19 has been reported worldwide. As the epidemiological scenario is rapidly changing daily, we still have no idea about the impact of COVID-19 in SSc. For this reason, data collection and sharing is of great importance. A registry of Italian Society of Rheumatology on rheumatic diseases and COVID-19 infection is now established, and the data will enter the international registry supported by America College of Rheumatology/European League Against Rheumatism and other rheumatologic societies worldwide. Very recently, an international task force under the WSF umbrella has released advices to help the practitioner when dealing with SSc patients [42].

In the very next future, a shared active scientific investigation will be fundamental to understanding the clinical implications of COVID-19 in SSc.

Code availability (software application or custom code) Not applicable.

Authors' contributions $\mathrm{MO}, \mathrm{GL}, \mathrm{CB}, \mathrm{MMC}$ conceived the presented idea. MO, MMC, LC, LZ, authors contributed to the final version of the manuscript. MMC, SBR, YV, AB, SG supervised the project.

Data Availability Not applicable.

Compliance with ethical standards Disclosures None.

\section{References}

1. Mahase E (2020) China coronavirus: WHO declares international emergency as death toll exceeds 200. BMJ 368:m408

2. https://covid19.who.int/ (Accessed 28 April 2020)

3. Cappelli S, Bellando Randone S, Camiciottoli G, de Paulis A, Guiducci S, Matucci-Cerinic M (2015) Interstitial lung disease in systemic sclerosis: where do we stand? Eur Respir Rev 24:411-419

4. Mihai C, Dobrota R, Schröder M, et al (2020) COVID-19 in a patient with systemic sclerosis treated with tocilizumab for SScILD Ann Rheum Dis; annrheumdis-2020-217442

5. D'Antiga L (2020) Coronaviruses and immunosuppressed patients. The facts during the third epidemic. Liver Transpl. March 20

6. Ceribelli A, Motta F, De Santis M et al (2020) Recommendations for coronavirus infection in rheumatic diseases treated with biologic therapy. J Autoimmun Apr 2:102442

7. Channappanavar R, Perlman S (2017) Pathogenic human coronavirus infections: causes and consequences of cytokine storm and immunopathology. Semin Immunopathol (5):39, 529-539

8. Xu M Xh, Li T, Sun W, et al (2020) Effective treatment of severe COVID-19 patients with tocilizumab. ChinaXiv; 20200300026

9. Blagojevic J, Legendre P, Matucci-Cerinic M et al (2019) Is there today a place for corticosteroids in the treatment of scleroderma? Autoimmun Rev 18(12):102403

10. EULAR guidance for patients during Covid-19 outbreak. https:// www.eular.org/eular_guidance_for_patients_covid19_outbreak. $\mathrm{cfm}$

11. ACR guidance for patients during Covid-19 outbreak. https://www. rheumatology.org/announcements. (Accessed 28 April 2020)

12. Bruni C, Praino E, Guiducci S, Bellando-Randone S, Furst DE, Matucci-Cerinic M (2017) Hydroxychloroquine and joint involvement in systemic sclerosis: preliminary beneficial results from a retrospective case-control series of an EUSTAR center. Joint Bone Spine 84(6):747-748 
13. Chauhan A, Tikoo A, AJHM T (2015) The enigma of the clandestine association between chloroquine and HIV-1 infection. HIV Med 16(10):585-590

14. Keyaerts E, Li S, Vijgen L, Rysman E, Verbeeck J, van Ranst M, Maes P (2009) Antiviral activity of chloroquine against human coronavirus OC43 infection in newborn mice. Antimicrob Agents Chemother 53(8):3416-3421

15. Vincent MJ, Bergeron E, Benjannet $\mathrm{S}$ et al (2020) Chloroquine is a potent inhibitor of SARS coronavirus infection and spread. Virol J 2(1):69

16. Wang M, Cao R, Zhang L, Yang X, Liu J, Xu M, Shi Z, Hu Z, Zhong W, Xiao G (2020) Remdesivir and chloroquine effectively inhibit the recently emerged novel coronavirus (2019-nCoV) in vitro. Cell Res 30(3):269-271

17. Gao J, Tian Z, Yang X (2020) Breakthrough: chloroquine phosphate has shown apparent efficacy in treatment of COVID-19 associated pneumonia in clinical studies. Biosci Trends 14(1):72

18. Chen J, Liu D, Liu L et al (2020) A pilot study of hydroxychloroquine in treatment of patients with common coronavirus disease-19 (COVID-19). Journal of ZheJiang University 49(1)

19. Cortegiani A, Ingoglia G, Ippolito $M$ et al (2020) A systematic review on the efficacy and safety of chloroquine for the treatment of COVID-19. J Crit Care S0883-9441(20):30390-30397

20. Ferner RE, Aronson JK (2020) Chloroquine and hydroxychloroquine in covid-19. BMJ 369:m1432

21. Gbinigie K, Frie K (2020) Should chloroquine and hydroxychloroquine be used to treat COVID-19? A rapid review. BJGP Open

22. CDC COVID-19 Response Team (2020) Preliminary estimates of the prevalence of selected underlying health conditions among patients with coronavirus disease 2019 - United States, February 12March 28, 2020. MMWR Morb Mortal Wkly Rep 69:382-386

23. Chen Y, Liu Q, Guo D (2020) Emerging coronaviruses: genome structure, replication, and pathogenesis. J Med Virol 92:418-423

24. Imai Y, Kuba K, Rao S, Huan Y, Guo F, Guan B, Yang P, Sarao R, Wada T, Leong-Poi H, Crackower MA, Fukamizu A, Hui CC, Hein L, Uhlig S, Slutsky AS, Jiang C, Penninger JM (2005) Angiotensinconverting enzyme 2 protects from severe acute lung failure. Nature 436:112-116

25. Goldin JG, Lynch DA, Strollo DC, Suh RD, Schraufnagel DE, Clements PJ, Elashoff RM, Furst DE, Vasunilashorn S, McNittGray M, Brown MS, Roth MD, Tashkin DP, Scleroderma Lung Study Research Group (2008) High-resolution CT scan findings in patients with symptomatic scleroderma-related interstitial lung disease. Chest 134:358-367

26. Suliman S, Al Harash A, Roberts WN et al (2017) Sclerodermarelated interstitial lung disease. Respir Med Case Rep 22:109-112

27. Launay D, Remy-Jardin M, Michon-Pasturel U, Mastora I, Hachulla E, Lambert M, Delannoy V, Queyrel V, Duhamel A, Matran R, de Groote P, Hatron PY (2006) High resolution computed tomography in fibrosing alveolitis associated with systemic sclerosis. J Rheumatol 33(9):1789-1801

28. Desai SR, Veeraraghavan S, Hansell DM, Nikolakopolou A, Goh NSL, Nicholson AG, Colby TV, Denton CP, Black CM, du Bois RM, Wells AU (2004) CT features of lung disease in patients with systemic sclerosis: comparison with idiopathic pulmonary fibrosis and nonspecific interstitial pneumonia. Radiology 232:560-567

29. Rodriguez-Morales AJ, Cardona-Ospina JA, Gutiérrez-Ocampo et al (2020) Clinical, laboratory and imaging features of COVID19: a systematic review and meta-analysis. Travel Med Infect Dis Mar 13:101623

30. Yang J, Zheng Y, Gou X et al (2020) Prevalence of comorbidities in the novel Wuhan coronavirus (COVID-19) infection: a systematic review and meta-analysis. Int J Infect Dis S1201-9712(20):3013630133

31. Chen N, Zhou M, Dong X, Qu J, Gong F, Han Y, Qiu Y, Wang J, Liu Y, Wei Y, Xia J', Yu T, Zhang X, Zhang L (2020) Epidemiological and clinical characteristics of 99 cases of 2019 novel coronavirus pneumonia in Wuhan, China: a descriptive study. Lancet 395(10223):507-513

32. Shi H, Han X, Jiang $N$ et al (2020) Radiological findings from 81 patients with COVID-19 pneumonia in Wuhan, China: a descriptive study. Lancet Infect Dis 20(4):425-434

33. Elhai M, Meune C, Boubaya M et al (2017) Mapping and predicting mortality from systemic sclerosis. Ann Rheum Dis 76(11):1897-1905

34. Elhai M, Meune C, Avouac J, Kahan A, Allanore Y (2012) Trends in mortality in patients with systemic sclerosis over 40 years: a systematic review and meta-analysis of cohort studies. Rheumatology (Oxford) 51(6):1017-1026

35. Li G, Fan YH, Lai YN, Fan Y, Lai Y, Han T, Li Z, Zhou P, Pan P, Wang W, Hu D, Liu X, Zhang Q, Wu J (2020) Coronavirus infections and immune responses. J Med Virol 92(4):424-432

36. Xie X, Zhong Z, Zhao W et al (2020) Chest CT for typical 2019nCoV pneumonia: relationship to negative RT-PCR testing. Radiology 12:200343

37. Huang P, Liu T, Huang L, Liu H, Lei M, Xu W, Hu X, Chen J, Liu B (2020) Use of chest CT in combination with negative RT-PCR assay for the 2019 novel coronavirus but high clinical suspicion. Radiology 295(1):22-23

38. Ai T, Yang Z, Hou H, et al (2020) Correlation of chest CT and RTPCR testing in coronavirus disease 2019 (COVID-19) in China: a report of 1014 cases. Radiology;200642

39. Fang Y, Zhang H, Xie J, et al (2020) Sensitivity of chest CT for COVID-19: comparison to RT-PCR. Radiology;200432

40. Faravelli C, Pietrini F, Rotella F et al (2019) Stressful life events at the onset and during the evolution of systemic sclerosis. Int J Clin Rheumatol 14(5):171-178

41. Del Rosso A, Boldrini M, D’Agostino D et al (2004) Health-related quality of life in systemic sclerosis as measured by the short form 36: relationship with clinical and biologic markers. Arthritis Rheum 51(3):475-481

42. Matucci Cerinic M, Bruni C, Allanore Y, et al (2020) Systemic sclerosis and the SARS2-COVID-19 virus pandemic: World Scleroderma Foundation preliminary advices for patient management. Ann Rheum Dis. pii: annrheumdis-2020-217407

Publisher's note Springer Nature remains neutral with regard to jurisdictional claims in published maps and institutional affiliations. 